\title{
Radiographier les volcans avec les rayons cosmiques
}

Dominique Gibert ${ }^{(1)}$ (gibert@ipgp.fr), Karine Marteau-Bazouni et Jacques Marteau ${ }^{2)}$ (marteau@ipnl.in2p3.fr) (1) Institut de Physique du Globe de Paris (UMR CNRS-INSU 7154), PRES Sorbonne Paris Cité, 1 rue Jussieu, 75238 Paris Cedex 05. (2) Institut de Physique Nucléaire de Lyon (UMR CNRS-IN2P3 5822), Université Lyon 1, 4 rue Enrico Fermi, 69622 Villeurbanne Cedex.

Les muons contenus dans

les gerbes de particules

produites par les rayons

cosmiques bombardant

en permanence la haute

atmosphère peuvent

traverser plusieurs kilomètres

de roches. La mesure de la

diminution de leur flux lors

de la traversée d'un volcan

permet d'en déterminer

la structure interne.

Il est ainsi possible

de radiographier les volcans

et d'en faire une

tomographie 3D, à l'instar

des scanners médicaux.

Remerciements

Les résultats présentés dans cet article sont le fruit de collaborations avec nos collègues de l'IPG de Paris (UMR 7154), l'IPN Lyon (UMR 5822), Géosciences Rennes (UMR 6118) et l'Observatoire Volcanologique et Sismologique de Guadeloupe. Les opérations de terrain ont bénéficié du concours de F. Dufour et des équipages de la base d'hélicoptère de la Sécurité Civile de Guadeloupe. Ces travaux sont financés par le CNRS/IN2P3 (programme interdisciplinaire Particules et Univers) et l'Agence Nationale de la Recherche (projet DomoScan). Contribution IPGP 3263.

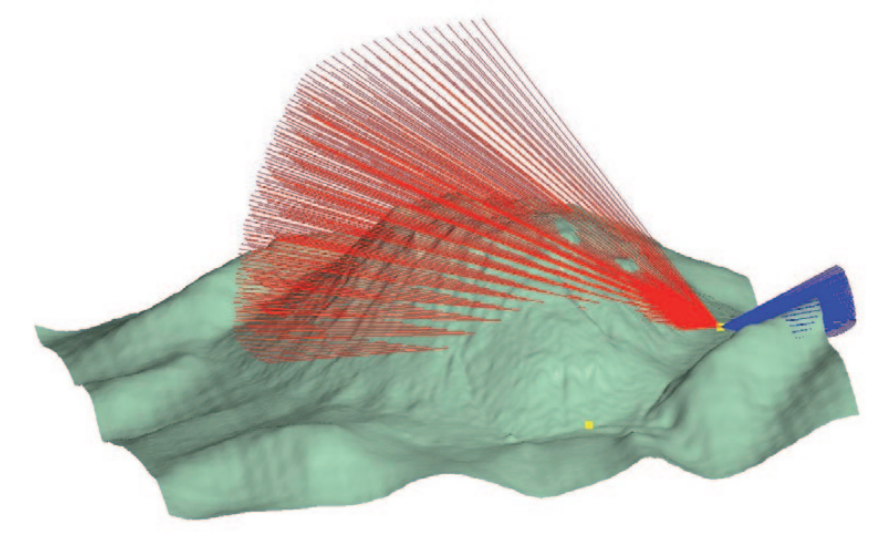

1. Principe de la radiographie par muons cosmiques. Un télescope placé en contrebas du volcan enregistre le flux de muons ayant traversé l'édifice volcanique. l'instrument offre une large couverture angulaire permettant de radiographier l'ensemble du volcan depuis un seul point de vue (rais rouges). La comparaison entre le flux émergeant du volcan et le flux à ciel ouvert permet de déterminer la quantité de matière rencontrée par les particules pendant leur trajet. Le télescope enregistre également le flux arrière (rais bleus).

Découvert en 1936 par Carl Anderson et Seth Neddermeyer au cours de leurs travaux sur les rayons cosmiques, le muon - également appelé électron lourd - est un lepton abondant dans les gerbes atmosphériques produites par les rayons cosmiques primaires. Au niveau du sol, le flux moyen de muons est d'environ $1 \mathrm{~cm}^{-2} \cdot \mathrm{min}^{-1}$ pour une énergie moyenne de $4 \mathrm{GeV}$. Cependant, tous les muons n'ont pas cette énergie, et celle-ci se distribue selon une loi de puissance (voir encadré 1).

Certains muons possèdent une énergie très importante - supérieure à $1000 \mathrm{GeV}$ - leur permettant de traverser plusieurs centaines de mètres de roche avant de se désintégrer. Ce sont ces particules très énergiques qui sont utilisées pour radiographier les volcans. Des télescopes comportant des matrices de détecteurs (voir encadré 2) sont placés en contrebas du volcan (fig. 1) pour compter et déterminer les trajectoires des muons possédant suffisamment d'énergie pour traverser le massif rocheux. En comparant le flux de muons ayant traversé le volcan au flux mesuré à ciel ouvert, il est possible de connaitre la quantité de matière que les muons ont rencontrée pendant leur traversée.

Connaître la structure interne des édifices volcaniques est d'une grande importance pour la surveillance des volcans dangereux et l'estimation des risques qu'ils représentent. Chauffés à leur base, les volcans tels que la Soufrière de Guadeloupe sont le siège d'une vigoureuse convection de fluides acides produits par la percolation des gaz magmatiques à travers les aquiferes rechargés par les abondantes pluies tropicales (fig. 2). La circulation hydrothermale qui en résulte est intense et affaiblit la résistance mécanique des dômes de lave tels que celui de la Soufrière de Guadeloupe. Cet affaiblissement peut placer le dôme en situation de déséquilibre et représente un risque important de déstabilisation, comme en témoigne l'histoire géologique de la région.

- Quels sont les volumes occupés par des matériaux altérés et instables?

- Quelle est l'extension des réservoirs hydrothermaux?

\〉 


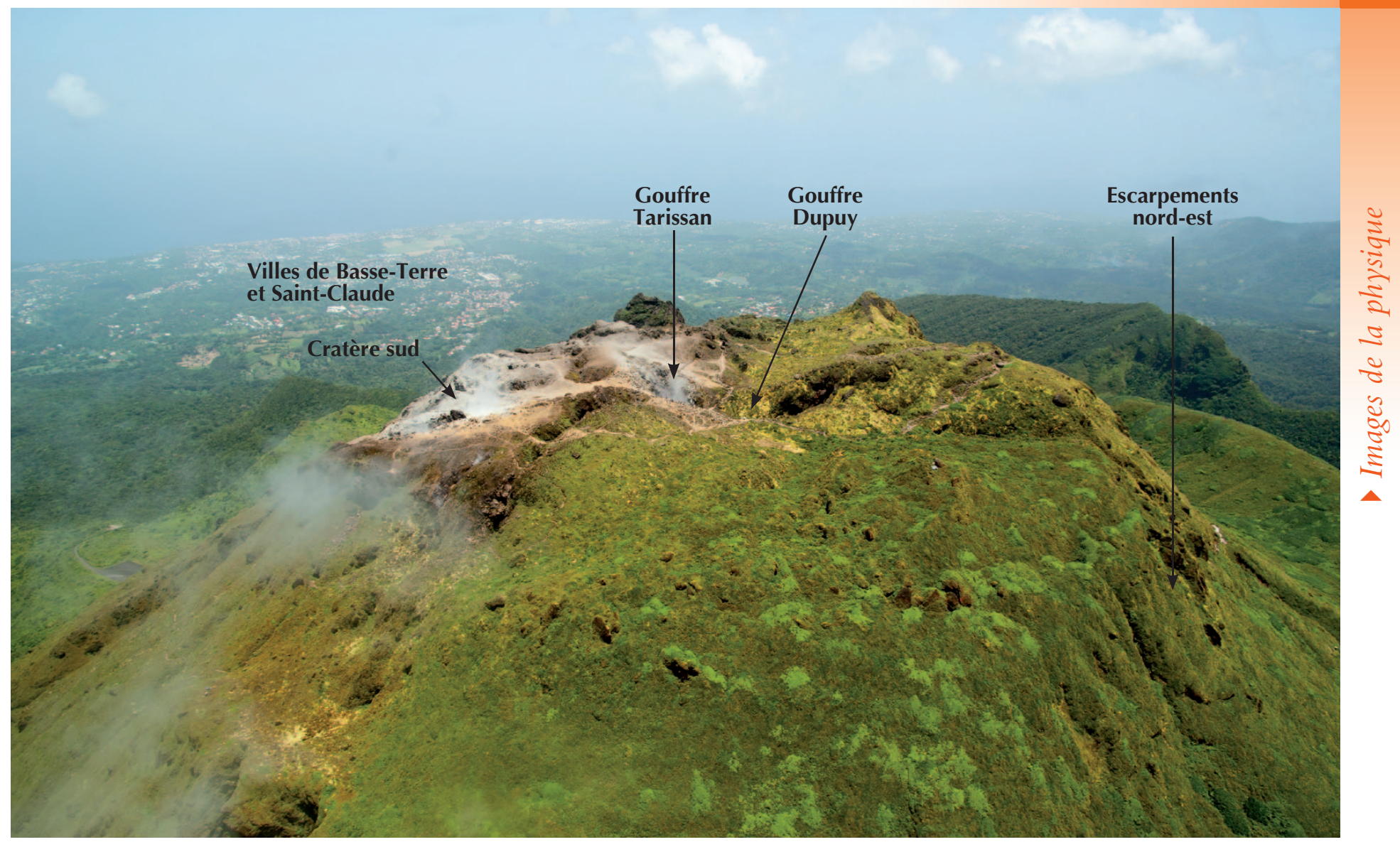

2. Vue vers l'ouest du dôme de la Soufrière de Guadeloupe. Dominant les villes de Basse-Terre et de Saint-Claude de ses 1467 mètres d'altitude, la Soufrière est le siège d'une intense activité hydrothermale dont les manifestations de surface sont concentrées dans le quart sud-est du plateau sommital. Cette photographie, prise le 4 juin 2012, montre la région du cratère sud correspondant à la zone peu dense 1 de la figure 5. Les escarpements situés au nord-est correspondent à la zone dense (4) située au nord de la radiographie. Les gouffres Tarissan et Dupuy sont à l’aplomb de la zone 2. (Cliché 0. Crispi, IPGP/OVSG).

\section{Les gerbes de particules et les muons}

Les muons $\mu^{+}$et $\mu^{-}$appartiennent à la catégorie des leptons, particules insensibles à l'interaction forte, à l'instar des électrons avec lesquels ils partagent de nombreux points communs. À tel point que le muon peut être considéré comme une " copie » de l'électron, environ 200 fois plus lourde $\left(\mathrm{m}_{u}=105 \mathrm{MeV} / \mathrm{c}^{2}\right)$ et surtout instable (le temps de vie d'un muon au repos est de 2,2 $\mu$ s). Les muons atmosphériques sont produits dans une chaîne d'interaction complexe initiée par les rayons cosmiques primaires. Ces derniers, essentiellement des protons et des noyaux légers, " bombardent » l'atmosphère terrestre et entrent en collision avec des noyaux d'oxygène ou d'azote. De cette première réaction complexe sont issues diverses particules. Parmi elles de nombreux pions $\pi^{ \pm}$et kaons $\mathrm{K}^{ \pm}$, particules instables se désintégrant dans le mode dominant suivant $: \pi / K^{+} \rightarrow \mu^{+}+v_{\mu}$.

Les muons ainsi produits sont ultra-relativistes, leur durée de vie est donc "boostée », ce qui explique qu'ils peuvent parcourir des distances importantes et atteindre la surface terrestre. Du fait de leur faible section efficace d'interaction avec la matière, les plus énergétiques d'entre eux peuvent même traverser des couches de roche importantes (jusqu'à une centaine de $\mathrm{GeV}$, un muon ne perd en moyenne qu'environ $2 \mathrm{MeV} / \mathrm{cm}$ à la traversée de la roche, ce qui lui permet de traverser des centaines de mètres ou plus). Il semble donc intéressant de les utiliser pour radiographier des structures larges.

Le flux moyen de muons cosmiques reçus à la surface terrestre est de l'ordre de 1 muon par $\mathrm{cm}^{2}$ et par minute. Les muons les plus énergétiques, et donc les plus pénétrants pour la radiographie, ont un flux inférieur de plusieurs ordres de grandeur à cette valeur moyenne, ce qui contraint les prises de vue en termes de durée et de sensibilité.

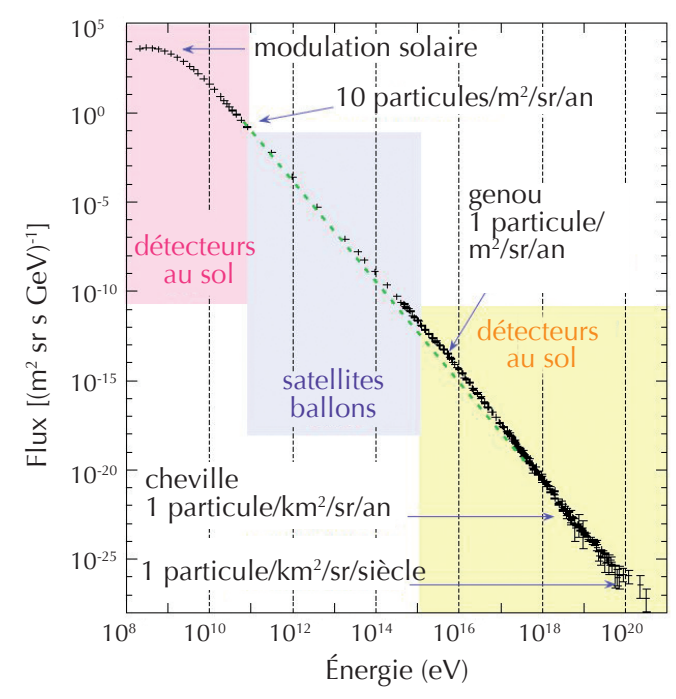

Le flux de muons cosmiques décroît rapidement avec l'énergie, suivant ainsi le spectre des cosmiques primaires (figure ci-dessus) dont ils sont issus, en $\mathrm{E}^{-(\gamma+1)}$, où $\gamma \sim 1,7$ à 2,0.

(Source: K. Kotera, thèse de doctorat, Université Pierre et Marie Curie). 


\section{La détection de particules chargées} encadré 2

La détection des particules chargées se fait au moyen d'un scintillateur plastique solide, un polystyrène dopé qui convertit en énergie lumineuse l'énergie perdue par chaque particule lors de sa traversée du détecteur (environ $2 \mathrm{MeV}$ par centimètre de plastique traversé, soit une petite fraction de l'énergie totale d'un muon cosmique moyen). Cette émission de photons, dans le bleu, se fait de manière isotrope à l'intérieur du barreau de scintillateur. Les parois du scintillateur sont recouvertes d'un réflecteur (à base de $\mathrm{TiO}_{2}$ ) pour maximiser la collecte des photons par une fibre optique collée au centre du barreau. Cette dernière, dite à décalage de longueur d'onde (Wave Length Shifting, WLS), absorbe les photons « bleus » émis par le scintillateur et réémet dans le jaune-vert, afin d'optimiser la réponse du photodétecteur à photocathode "bi-alkali ». Le schéma suivant illustre le principe de détection.

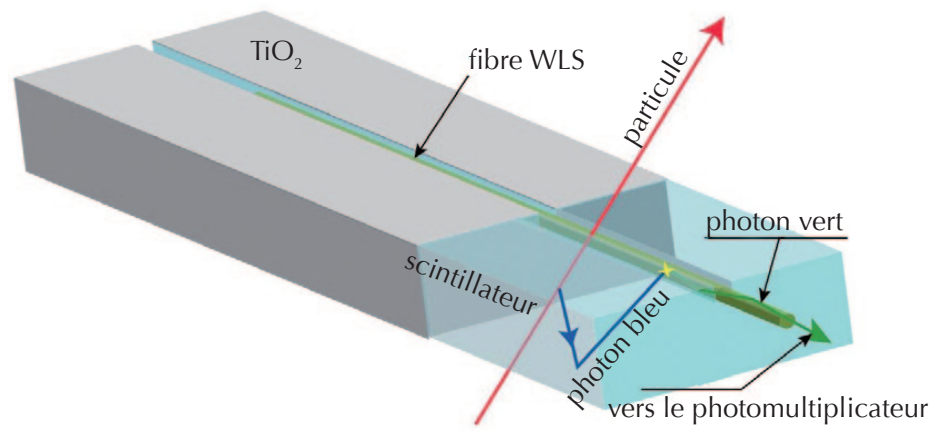

On réalise une connexion optique de chaque fibre vers une fibre optique "claire ", à spectre plat, pour déporter, sans atténuation, le signal vers un pixel d'un photodétecteur unique, placé au centre de la matrice et vers lequel convergent l'ensemble des 32 fibres assurant la lecture de 16 barreaux dans la direction $\mathrm{X}$ et de 16 barreaux dans la direction orthogonale $Y$. Une matrice de détection contient deux plans de détection, $X$ et $Y$, de $80 \mathrm{~cm}$ de côté (photographie ci-dessous).

Ainsi, lorsqu'une particule traverse la matrice de détection, son énergie déposée est convertie en une coordonnée $(X, Y)$ correspondant à un couple de pixels du photomultiplicateur. Les (au moins) deux canaux touchés génèrent un signal de déclenchement intelligent qui lance la séquence de lecture des signaux intéressants (temps de l'événement, nombre total de canaux, énergie déposée dans le scintillateur) puis leur numérisation, formatage et transfert vers un processeur central qui collecte les données de l'ensemble des matrices de détection du télescope (3 au minimum). Toutes les données qui coïncident dans une même fenêtre temporelle constituent un " événement » comportant une ou plusieurs trajectoire(s) de particule(s).

Le principe de détection et sa mise en œuvre pratique reproduisent en miniature le trajectographe de l'expérience OPERA (Oscillation Project with Emulsions tRacker Apparatus ${ }^{(a)}$ ), installée dans le laboratoire souterrain du Gran Sasso en Italie, et vouée à l'étude des propriétés des neutrinos générés artificiellement au CERN à Genève à 732 km de distance.

(a) http://operaweb.Ings.infn.it

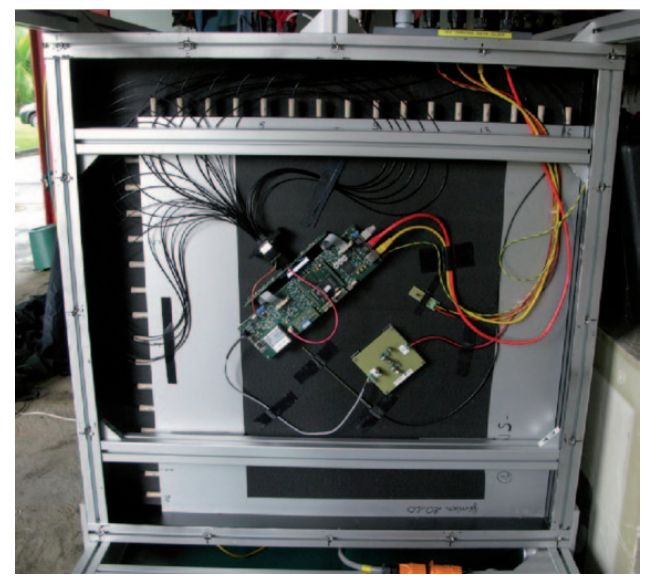

Vue d'une matrice de détection comprenant deux séries perpendiculaires de 16 barreaux qui définissent 256 pixels.

\section{$\gg\rangle$}

- Quelle est l'énergie thermique qu'ils contiennent et qui peut être libérée lors d'une crise éruptive ?

- Quel est le confinement de ces réservoirs pouvant conduire à une dangereuse mise sous pression?

- Où se situe l'interface liquide-vapeur?

- Quel est le contenu en gaz et le potentiel explosif du conduit d'alimentation des dômes de lave en croissance comme celui de la Soufrière Hills de Montserrat ?

Voici des questions importantes auxquelles le projet DIAPHANE de tomographie par muons, né d'une collaboration entre géophysiciens et physiciens des particules, tente d'apporter des réponses.

Les avantages de la tomographie par muons sont multiples. Le premier d'entre eux est que cette méthode permet de déterminer la masse volumique des roches d'une manière analogue à celle utilisée en tomographie médicale, c'est-à-dire à partir de mesures donnant la densité moyenne le long des lignes de visée du télescope (en rouge sur la figure 1).

Un second avantage de la tomographie par muons est de permettre de réaliser une tomographie à distance, ce qui est une possibilité très intéressante pour l'étude des volcans en éruption tels que la Soufrière Hills de Montserrat, placée sous la surveillance des équipes de l'IPG de Paris.

\section{Les télescopes: principe de détection}

Les télescopes (fig. 3) utilisent des matrices de détection formées de lattes de matériau qui scintille à la traversée de particules ionisantes, telles que des muons et des électrons (voir encadré 2). Les trois matrices du télescope, qui comprennent chacune 256 pixels, permettent alors de déterminer trois points $(\mathrm{X}, \mathrm{Y})$ de traversée de ces particules, ce qui suffit pour reconstruire leurs trajectoires avec une certaine redondance. L'ensemble couvre ainsi 961 lignes de visée $\mathbf{r}_{\mathrm{m}, \mathrm{n}}$ (fig. 1). Ces dernières forment un cône dont l'ouverture peut être ajustée en réglant l'écartement entre les matrices. Cet écartement, ainsi que la surface utile des matrices, définissent les capacités de détection de l'instrument représentées par l'acceptance $\mathcal{T}^{\prime}$ (en $\mathrm{cm}^{2}$.sr), telle que (fig. 4) :

$v\left(\mathbf{r}_{\mathrm{m}, \mathrm{n}}, \Delta T\right)=I\left(\mathbf{r}_{\mathrm{m}, \mathrm{n}}\right) \times \Delta T \times \mathcal{T}\left(\mathbf{r}_{\mathrm{m}, \mathrm{n}}\right)$ 
où $v$ est le nombre de muons détectés dans l'intervalle de temps $\Delta T$ pour la ligne de visée $\mathbf{r}_{\mathrm{m}, \mathrm{n}}$ et $I\left(\mathrm{en} \mathrm{cm}^{-2} \cdot \mathrm{sr}^{-1} \cdot \mathrm{s}^{-1}\right)$ est le flux de muons qui traverse le volcan dans la direction concernée. L'estimation du flux $I\left(\mathbf{r}_{\mathrm{m}, \mathrm{n}}\right)$ à partir du nombre de particules détectées $v\left(\mathbf{r}_{\mathrm{m}, \mathrm{n}}, \Delta T\right)$ suppose une connaissance précise de la fonction d'acceptance $\mathcal{T}\left(\mathbf{r}_{\mathrm{m}, \mathrm{n}}\right)$. En pratique, cette fonction est déterminée expérimentalement sur le terrain en effectuant une brève séquence de mesure à ciel ouvert, à partir de laquelle il est possible d'estimer l'efficacité des barres de scintillateur constituant les matrices. Cette calibration est faite périodiquement et à chaque mise en place du télescope.

L'équation (1) peut être utilisée pour définir le domaine de fonctionnement du télescope. Par exemple, si l'on souhaite distinguer des anomalies de densité $\delta \rho$ au sein d'un volcan de densité $\rho_{0}$, il est nécessaire de distinguer de façon significative $v\left(\rho_{0}, \Delta T\right)$ de $v\left(\rho_{0}+\delta \rho, \Delta T\right)$. En adoptant une statistique de Poisson pour $\mathrm{v}$, on peut montrer que l'anomalie de densité $\delta \rho$ est décelable si :

$\Delta T \times \mathcal{T}^{\prime} \times\left[\Delta I^{2}\left(\rho_{0}, \delta \rho\right) / I\left(\rho_{0}\right)\right]>\alpha$

où la constante $\alpha$, typiquement comprise entre 1 et 2, fixe le rapport signal sur bruit désiré. $\Delta I$ est la variation de flux produite par la variation de densité $\delta \rho$ au sein du massif rocheux de densité $\rho_{0}$. La formule de faisabilité (2) est d'un grand intérêt pratique, car elle permet de dimensionner les expériences en fonction des objectifs scientifiques à atteindre. L'acceptance, qui intervient dans l'équation ci-dessus, dépend de l'angle solide couvert par chaque ligne de visée de l'instrument. Une résolution spatiale élevée implique donc une acceptance réduite et nécessite en conséquence une plus longue durée d'acquisition. Par exemple, le télescope de la figure 3 permet d'obtenir une radiographie complète de la Soufrière de Guadeloupe avec une résolution d'environ 20 mètres (fig. 5) en un peu plus d'un mois. L'étude de variations temporelles de densité nécessite, quant à elle, une grande acceptance, qui peut être obtenue en installant plusieurs télescopes en réseau afin de constituer un instrument composite aux capacités de détection accrues. Cette mise en réseau est rendue possible grâce à l'utilisation des systèmes d'acquisition mis au point pour l'expérience OPERA, destinée à l'étude des neutrinos.

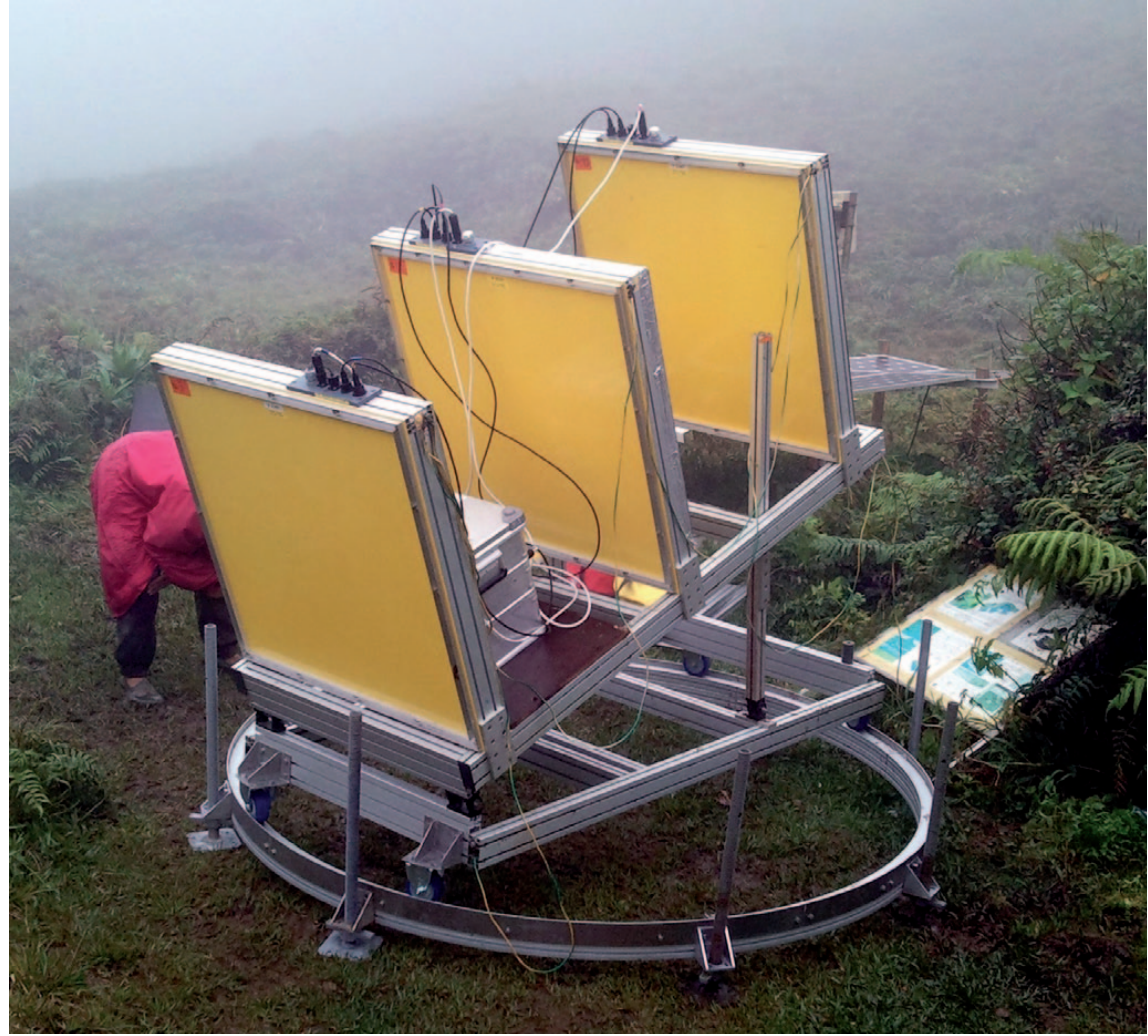

3. Un télescope sur la Soufrière de Guadeloupe. Les trois cadres parallèles (de couleur jaune) contiennent les matrices de détection. La matrice centrale peut être équipée de blindages d'acier de $25 \mathrm{~mm}$ d'épaisseur. Équipé d'un vérin et d'une base circulaire, l'instrument est facilement orientable. Lorsqu'il est en opération, il est muni de protections contre les pluies et les vents forts qui règnent sur le volcan.

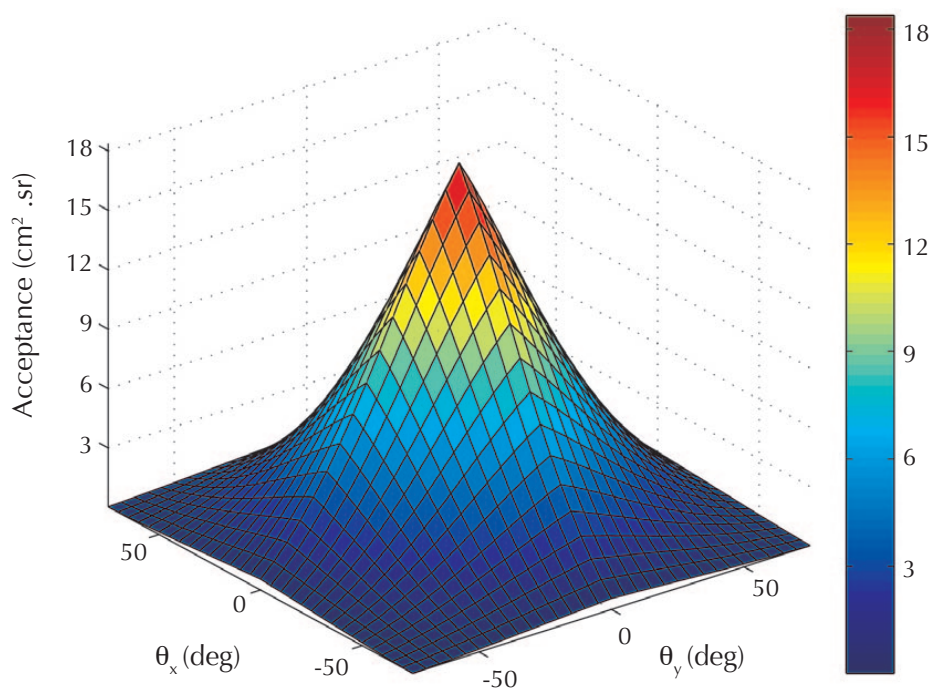

4. Acceptance $\mathcal{T}$ du télescope. Elle prend sa valeur maximale pour la ligne de visée perpendiculaire aux matrices et tend vers zéro pour les lignes très obliques. 


\section{Les radiographies}

Les radiographies obtenues pour la Soufrière de Guadeloupe (fig. 5) révèlent la structure hétérogène de ce volcan soumis à une intense activité hydrothermale depuis son apparition, il y a un peu moins de cinq siècles. Le dôme de la Soufrière donne l'apparence d'une "dent creuse ", dont la partie centrale est occupée par des zones de faible densité (notées 1 et 2 sur la figure) formées de matériaux altérés et peu consolidés. Les mesures réalisées avec le télescope donnent la densité intégrée le long des lignes de vue (en rouge sur la figure 1), et un modèle topographique du volcan a été utilisé pour obtenir la radiographie de la figure 5 qui représente la densité moyenne le long de ces lignes.

Le compartiment peu dense noté 1 sur la figure 5 correspond au cratère sud, où se trouvent actuellement les fumerolles les plus actives du volcan. Celles-ci émettent une vapeur extrêmement acide, qui témoigne de la présence de fluides corrosifs à l'origine d'une altération intense des roches. La faible densité de cette zone reflète également la présence de nombreuses fractures visibles en surface.

La grande zone de faible densité notée 2 sur la figure, est située sous une ligne de fractures et de gouffres (Tarissan et Dupuy) ayant fait l'objet d'une activité intense lors des éruptions phréatiques qui ont ponctué l'histoire du dôme. Là encore, la faible densité de cette zone reflète vraisemblablement la présence de cavités importantes telles que la grotte Spallanzani, inaccessible depuis le milieu du $19^{\text {e }}$ siècle, et décrite comme une succession de trois vastes salles s'étendant du flanc nord du dôme jusqu'à son centre. Cette zone peu dense semble se prolonger en profondeur.

Contrairement à la zone hydrothermale du cratère sud (1) qui apparaît non confinée, la zone (2) est surmontée d'une barrière plus dense (3), susceptible de constituer un

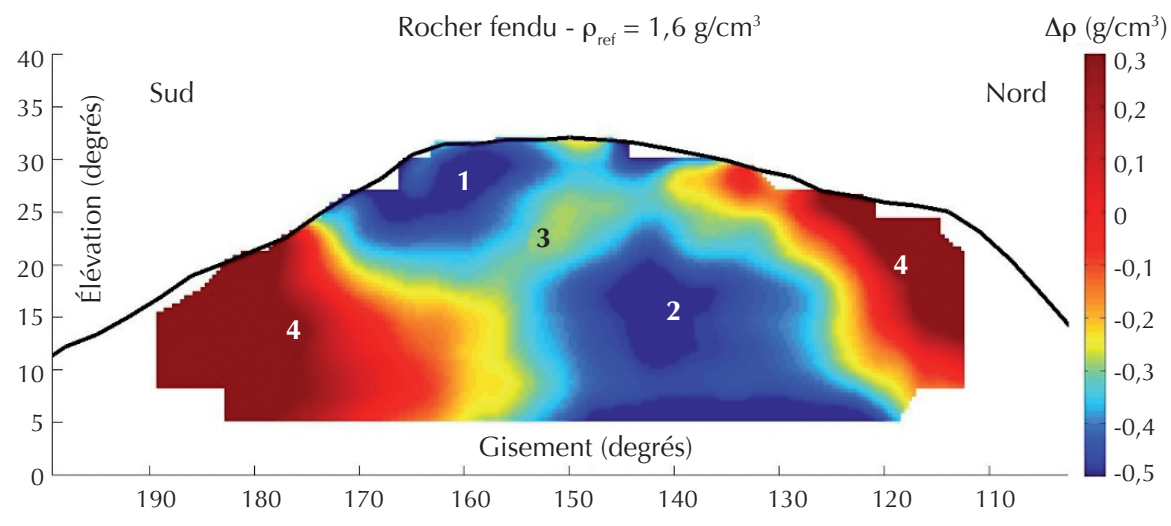

5. Radiographie de la densité moyenne de la Soufrière de Guadeloupe selon un plan nord-sud. La radiographie, obtenue sur le site dit de la roche fendue, met en évidence l'allure de « dent creuse » du dôme avec des zones centrales peu denses (par rapport à la densité de référence $\left.\rho_{\text {ref }}=1,6 \mathrm{~g} / \mathrm{cm}^{3}\right)$ (1 et 2) formées de matériaux altérés, entourées de zones latérales denses (4). La zone de densité intermédiaire (3) peut constituer un couvercle permettant la montée en pression de la zone hydrothermale (2). L'angle de gisement (ou d'azimut) est compté depuis la direction est. La résolution spatiale de l'image obtenue est de l'ordre de 20 mètres. Voir également la figure 2.

couvercle permettant la montée en pression du réservoir hydrothermal et d'éventuels épisodes explosifs en cas de surchauffe du volcan. La réalisation de radiographies sous d'autres angles de vue permettra de préciser le degré de confinement du système et de repérer de possibles zones de faiblesse dans l'auréole dense (4) qui confine latéralement le réservoir.

\section{Perspectives}

La combinaison des radiographies réalisées sous des angles différents permettra d'obtenir une vue tridimensionnelle des hétérogénéités de densité au sein du dôme de lave. Ce type de modèle structural apportera des contraintes sur les modèles de déstabilisation. La reconstruction tridimensionnelle consiste à poser un système d'équations reliant la distribution de densité recherchée aux densités moyennes le long des lignes de visée du télescope (fig. 1). Même si la résolution de ce système d'équations reste un problème mal posé à cause du petit nombre d'angles de vue disponibles, elle est plus stable que la résolution du difficile problème inverse rencontré en gravimétrie, où les hétérogénéités de densité sont reconstruites à partir de mesures du champ de pesanteur.

Plusieurs modélisations interviennent dans la longue chaîne de traitements conduisant du nombre de muons détectés à la distribution 3D de densité du volcan : flux à ciel ouvert, acceptance du télescope, modèle topographique, etc. Nous avons adopté une formulation probabiliste afin de coupler ces différents modèles dans un cadre bayésien où la solution a posteriori d'un modèle constitue l'a priori du modèle suivant.

La tomographie par muons vient très utilement compléter la panoplie des méthodes d'imagerie géophysique en permettant la détermination directe de la densité de structures géologiques fortement hétérogènes, souvent difficiles à visualiser par d'autres méthodes. Les volcans sont des objets géologiques particulièrement adaptés à l'application de cette nouvelle méthode, dont l'utilisation devrait rapidement s'étendre.

\section{En savoir plus}

- M. Crozon, Quand le ciel nous bombarde, Vuibert (2005).

- J. Dubois et J.-L. Le Mouël, Volcans actifs français et risques volcaniques: Martinique, Guadeloupe, Réunion, Pacifique, Dunod Universciences (2007).

- M. Feuillard, La Soufrière de la Guadeloupe : un volcan et un peuple, Jasor (2011).

- D. Gibert, F. Beauducel, Y. Déclais, N. Lesparre, J. Marteau, F. Nicollin et A. Tarantola, "Muon Tomography: Plans for Observations in the Lesser Antilles", Earth Planets and Space, 52 (2010) 153-165.
- J.-C. Komorowski, Y. Legendre, B. Caron et G. Boudon, "Reconstruction and analysis of sub-plinian tephra dispersal during the 1530 A.D. Soufrière (Guadeloupe) eruption : Implications for scenario definition and hazards assessment", J. Volcanol. Geoth. Res., 178 (2008) 491-515.

- C. Lachaud, T. Beau et E. Armengaud, « L'observatoire Pierre Auger à la quête des énergies extrêmes ", Images de la Physique, CNRS Éditions (2006) 35-40.
- N. Lesparre et al., "Geophysical muon imaging: feasibility and limits”, Geophysical Journal International, 183 (2010) 1348-1361.

- J. Marteau et al., "Muons tomography applied to geosciences and volcanology", Nuclear Instruments and Methods in Physics A, 695 (2012) 23-28.

- J. Paul et J.-L. Robert-Esil, Le roman des rayons cosmiques, Ellipses (2009).

- F. Vannucci, Le vrai roman des particules élémentaires, Dunod (2010) 